\title{
Oncogene-mediated tumor transformation sensitizes cells to autophagy induction
}

\author{
RICARDO GARGINI $^{1-3}$, VEGA GARCÍA-ESCUDERO ${ }^{1,3}$, MARTA IZQUIERDO ${ }^{1}$ and FRANCISCO WANDOSELL ${ }^{1,3}$ \\ ${ }^{1}$ Centro de Biología Molecular Severo Ochoa (CSIC-UAM), Universidad Autonoma 28049 Madrid; \\ ${ }^{2}$ Centro Nacional de Biotecnología (CNB-CSIC), 28049 Madrid; ${ }^{3}$ Centro de Investigación Biomédica en Red \\ de Enfermedades Neurodegenerativas (CIBERNED), 28031 Madrid, Spain
}

Received March 13, 2015; Accepted May 18, 2015

DOI: $10.3892 /$ or.2016.4699

\begin{abstract}
The process of tumorigenesis induces alterations in numerous cellular pathways including the main eukaryotic metabolic routes. It has been recently demonstrated that autophagy is part of the oncogene-induced senescence phenotype although its role in tumor establishment has not been completely clarified. In the present study, we showed that non-transformed cells are sensitized to mitochondrial stress and autophagy induction when they are transformed by oncogenes such as $c-M y c$ or Ras. We observed that overexpression of $c-M y c$ or Ras increased AMP-activated protein kinase (AMPK) phosphorylation and the expression of p62, a known partner for degradation by autophagy. The activation of AMPK was found to favor the activation of FoxO3 which was prevented by the inhibition of AMPK. The transcriptional activation mediated by FoxO3 upregulated genes such as BNIP3 and LC3. Finally, the transformation by oncogenes such as $c-M y c$ and Ras predisposes tumor cells to autophagy induction as a consequence of mitochondrial stress and impairs tumor growth in vitro and in vivo, which may have therapeutic implications.
\end{abstract}

\section{Introduction}

Macroautophagy, also known as autophagy, is a catabolic process that operates by generating several benefits for the cell, protecting it against multiple stress stimuli. In the majority of cases, autophagy functions as a recycling and recovery system for proteins, lipids and DNA damage; therefore, the cell eliminates various compounds that may be toxic, and also generates ATP. It has been reported that autophagy protects the cell against DNA damage, chromosomal instability and metabolic

Correspondence to: Professor Francisco Wandosell, Centro de Biología Molecular Severo Ochoa, (CSIC-UAM), Universidad Autónoma de Madrid, Nicolás Cabrera 1, Cantoblanco 28049, Madrid, Spain

E-mail: fwandosell@cbm.uam.es

Key words: autophagy, RAS, mitochondrial stress, AMPK, FoxO stress (1). The exogenous expression of a great variety of oncogenes leads to tumorigenesis which is often accompanied by metabolic changes. In this aspect, one of the better examples is aneuploid tumors, where the additional chromosomes bear an increased amount of various proteins, affecting protein folding and turnover. The overaccumulation of proteins induces the activation of adaptive processes to alleviate this proteotoxic effect, such as the increase in chaperone Hsp72, the activation of AMPK or the degradation mediated by autophagy (1). These alterations of the metabolism in this type of tumor offer the possibility of blocking autophagy or generating metabolic stress as a therapeutic strategy due to the differential sensitivity of cancer cells (2).

Numerous oncogenic processes favor adaptations of signaling pathways to allow tumor transformation, although they generate cellular stress. This affects the autophagic pathway since in the majority of stress conditions the cells activate several signaling pathways, such as mTOR, AMPK, p53, JNK, FoxO3 among others, which regulate, positively or negatively, the induction of autophagic vacuoles. Most of these pathways control the expression of proteins related to vacuole generation, such as the Atgs. In contrast, both p53 and FoxO3 have been found to induce genes such as Sestrin 2 and DRAM or BNIP3 and GABARAP, respectively. However, various functions of these genes have not been completely elucidated since they are only associated with an increased degradative phase and in some circumstances with cell death.

During the Ras-mediated process of oncogenesis, there is an induction of autophagy through negative feedback in which Ras-Raf activation shrinks the PI3K pathway, reducing AKT phosphorylation (3). Therefore, this decrease in Akt activity reduces FoxO repression and permits the upregulation of protein, such as LC3, BNIP3 and ULK (4). In this manner, RAS-v12 overexpression induces an increase in autophagy that is associated with oncogene-induced senescence (OIS) hallmarks and also with the formation of TOR-autophagy spatial coupling compartment (TASCC) that mediates the secretion of interleukin-6/8, which are involved in senescence $(5,6)$. If once transformation is established and the stress situation is maintained, this generates autophagy dependence or an increase in the glucose uptake as previously demonstrated $(7,8)$.

In contrast, the involvement of AMPK in the regulation of metabolism and autophagy has been widely demonstrated. 
This kinase performs several functions that link metabolism and proliferation. In addition to its function as a sensor of the ATP/AMP ratio, this kinase is activated in response to oxidative and proteolytic stress.

In this sense, it has been recently reported that AMPK directly phosphorylates FoxO3 in its Ser413 and Ser588 residues, increasing the transcription of various $\mathrm{FoxO} 3$ regulated genes, such as Gadd45 $\alpha$ (9). Thus, much of the metabolic adaptation in cells which undergo oncogenic transformation may induce a differential response of AMPK depending on ATP levels, oxidative stress and autophagy.

In the present study, we demonstrated that the overexpression of c-Myc or RAS-v12 in untransformed mammary cells activates AMPK and that the phosphorylation of FoxO3 increases the transcription of genes such as BNIP3. Using a powerful system of mitophagy induction, the linamarase/ linamarin/glucose oxidase system (lis/lin/GO) $(10,11)$, we demonstrated that c-Myc or RAS-v12 transformation sensitized cells to autophagy, in vitro and in vivo.

\section{Materials and methods}

Reagents and antibodies. The antibody against FoxO3 P-ser413 was a kind gift from Dr Anne Brunet (Department of Genetics, Stanford University, Stanford, CA, USA). The commercial primary antibodies used were: FoxO3 and phospho-AMPKa (Thr172) (Cell Signaling, Danvers, MA, USA); pan-RAS (Calbiochem, Darmstadt, Germany); p62 (BD Biosciences, Bedford, CA, USA); E-cadherin and c-Myc (sc-788) (Santa Cruz Biotechnology, Santa Cruz, CA, USA); LC-3, BNiP3 and actin (Sigma, St. Louis, MO, USA). The secondary antibodies for western blot analysis were horseradish peroxidase-conjugated anti-rabbit or anti-mouse IgGs (Santa Cruz Biot'echnology). The reagents used were: linamarin (lin; $500 \mu \mathrm{g} / \mathrm{ml}$; Toronto Research Chemicals, Toronto, Canada), glucose oxidase (GO; 5-5.5 mEU/ml), 3-methyladenine (3MA; $10 \mathrm{mM}$ ) and thiazolyl blue tetrazolium bromide (MTT; $200 \mu \mathrm{g} / \mathrm{ml}$ ) (all from Sigma), bafilomycin A1 and compound $\mathrm{C}(10 \mu \mathrm{M})$ (from Calbiochem).

Media and cell culture. The MCF7 cell line was cultured in Dulbecco's modified Eagle's medium (DMEM) (Gibco, Life Technologies, Barcelona, Spain) supplemented with $10 \%$ fetal calf serum (FCS) at $37^{\circ} \mathrm{C}$ in $7 \% \mathrm{CO}_{2}$ and $97 \%$ relative humidity. The MCF10A cell line was grown as recommended by Joan S. Brugge (Whitehead Institute for Biomedical Research, Cambridge, MA, USA) in DMEM:F12 media (1:1) supplemented with $10 \mu \mathrm{g} / \mathrm{ml}$ insulin (Gibco), $20 \mathrm{ng} / \mathrm{ml}$ EGF (Tocris, Bristol, UK), $0.5 \mu \mathrm{g} / \mathrm{ml}$ hydrocortisone (Calbiochem), $100 \mathrm{ng} / \mathrm{ml}$ cholera toxin and $5 \%$ horse serum at $37^{\circ} \mathrm{C}$ in $7 \%$ $\mathrm{CO}_{2}$ and $97 \%$ relative humidity.

Adenovirus, lentivirus and retrovirus production. Adenolis vectors were obtained from Crucell (Leiden, The Netherlands). Pseudotyped lentivectors were produced using reagents and protocols from Didier Trono with the following modifications: $293 \mathrm{~T}$ cells were transiently co-transfected with $5 \mu \mathrm{g}$ of the corresponding lentivector plasmid, $5 \mu \mathrm{g}$ of the packaging plasmid pCMVdR8.74 and $2 \mu \mathrm{g}$ of the VSV-G envelope protein plasmid pMD2G using Lipofectamine Plus reagent following instructions of the supplier (Invitrogen, Life Technologies, Barcelona, Spain). The lentivectors included pMIG-h-c-Myc, that was kindly provided by Maria Soengas (CNIO, Madrid, Spain) (12), and pLenti-RAS-v12 provided by Judith Campisi (Lawrence Berkeley National Laboratory, Berkeley, CA, USA) (13) which encode human c-Myc and constitutively active RAS, respectively. To generate the different MCF10A derivative cells, the retroviral vectors used were pBabe, pBabe-dnFoxO3 and pBabe-FoxO3A (caFoxO3) that were kindly provided by Dr Clemens Schmitt (Max Delbrück Center for Molecular Medicine, Berlin, Germany). The retrovirus supernatant was prepared by transfection of phoenix-Ampho cells (Garry Nolan; http://www.stanford.edu/group/nolan/) with $5 \mu \mathrm{g}$ of each plasmid with Lipofectamine Plus as previously mentioned, and infected cells were selected with $0.5 \mu \mathrm{g} /$ $\mathrm{ml}$ of puromycin.

Soft agar assay. To evaluate the tumorigenic potential, $2 \times 10^{4}$ viable cells/well were plated in soft agar in 6-well plates. Briefly, the base layer was constructed by mixing equal agar and $2 \mathrm{X}$ medium, to obtain a final solution of $0.5 \%$ agar in 1X DMEM:F12 medium and for the top layer, the agar was diluted to $0.7 \%$ in distilled water with $2 \mathrm{X}$ DMEM medium. The cells were immediately added to the mix to yield a final solution of $0.35 \%$ agar in 1X DMEM medium, containing 30,000 cells $/ \mathrm{ml}$. The cells were grown for 10 days at $37^{\circ} \mathrm{C}$ in a humidified atmosphere containing $5 \% \mathrm{CO}_{2}$, and viable colonies were then stained with $1 \mathrm{ml} /$ well of $600 \mu \mathrm{g} / \mathrm{ml} \mathrm{MTT} \mathrm{for}$ $2 \mathrm{~h}$ and then were photographed.

Immunoblot analysis. Cell lysates were prepared by extracting proteins with lysis buffer $(50 \mathrm{mM}$ pH 7.5 Tris- $\mathrm{HCl}, 300 \mathrm{mM}$ $\mathrm{NaCl}, 0.5 \% \mathrm{SDS}$ and $1 \%$ Triton X-100). Proteins were separated by SDS-PAGE and transferred to nitrocellulose membranes (Bio-Rad, Hercules, CA, USA). The blots were developed using peroxidase-conjugated secondary antibody, and proteins were visualized using an enhanced chemiluminescence-based detection kit (Pierce, Rockford, IL, USA).

Mice and treatments. Nude mice, BALB/c nu/nu, were inoculated subcutaneously in both flanks with $1 \times 10^{6}$ MCF7-Ras-lis cells ( $n=6 /$ group). After 5 days, the animals were intratumorally treated daily with lin $(5 \mathrm{mg})$ and GO (2 EU) in the right tumor while the left one was left untreated. All treatments were performed under general anesthesia with isofluorane (AP/DRUGS/220/96; Baxter, Valencia, Spain). Tumor size (length, width and height) was measured when indicated with a caliper, and the tumor volume $(\mathrm{V})$ was calculated as: $\mathrm{V}=$ [ $\pi / 6$ (length $\mathrm{x}$ width $\mathrm{x}$ height)]. For animal care and handling we followed the Spanish legislation and guidelines (Spanish Royal Decree 1201/2005 BOE published on October 21, 2005), and those from the European Union (2003/65/CE from the European Parliament and Council July 2003) and those of the CBMSO Institutional Biosafety Committee.

Statistical analysis. Statistical comparison of the data groups was carried out using the Student's t-test. The differences are expressed with their corresponding statistical significance or p-value, which is the probability that the observation in a sample occurred merely by chance under the null hypothesis. 

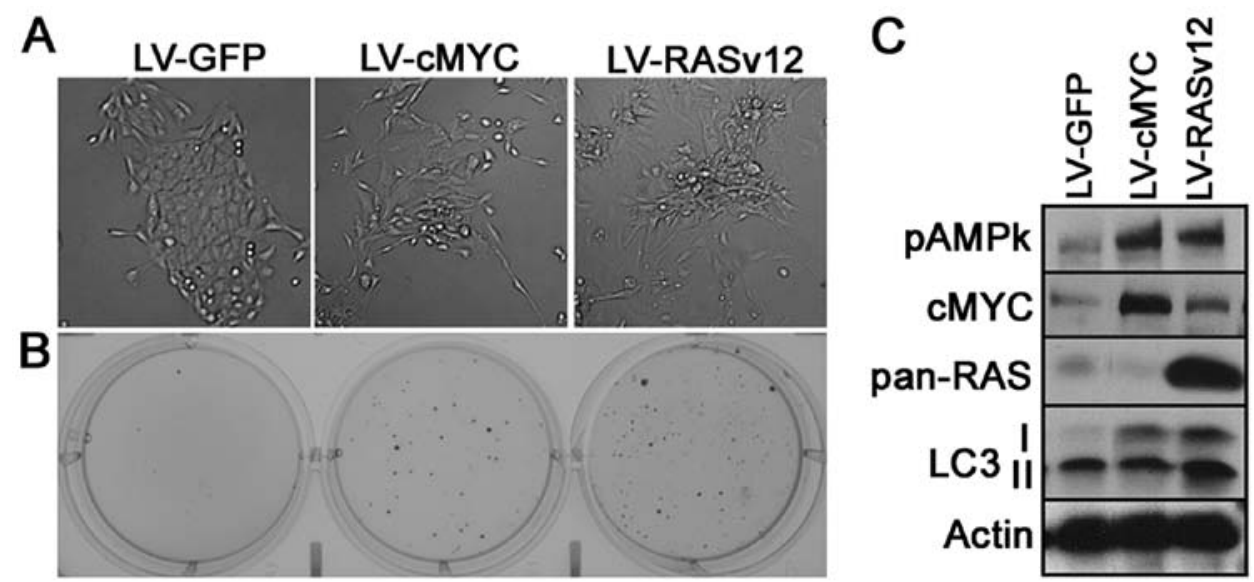

Figure 1. Tumor transformation by oncogenes increases the level of LC3-II. (A) MCF10A cells were infected with a lentivector encoding GFP or c-Myc or RASv12, and then representative bright field images were captured. (B) Colony formation assay of cells expressing GFP, c-Myc, or Ras-V12. (C) Representative western blot analysis of MCF10A cells expressing GFP (control), c-Myc or RASv12. The western blot analyses showing levels of LC3-II and phospho-AMPK (Thr172); and the corresponding transgenes c-Myc and Ras. Actin was used as a loading control.

\section{Results}

The purpose of the present study was to verify whether oncogene-mediated transformation sensitizes cells to autophagy. For this purpose we used two alternative approaches, the transfection of c-Myc $(14,15)$ or a mutant version of ras (RASv12) (16), and we initially used MCF10A, an untransformed mammary cell line, as a cell model. Next, we transfected and generated cell lines expressing either c-Myc or RASv12 (HrasVal12) or an empty vector containing GFP as an internal control. The c-Myc- and RASv12-expressing cells were characterized at three levels. First they were phenotypically altered giving the appearance of a mesenchymal-like phenotype (Fig. 1A). Second, both oncogenic transformations conferred MCF10A cells the ability of anchorage-independent growth forming colonies in soft agar when compared with the mock-transfected cells (LV-GFP) (Fig. 1B). Third, they expressed and maintained a high level of either c-Myc or RAS as confirmed by western blotting (Fig. 1C).

As an initial indicator of autophagic vesicles, we determined the level of LC3-II. We showed that c-Myc-transformed cells, while more prominent in Ras-transformed cells, showed an increase in the amount of total LC3B, as well as an increase in the LC3-II form. Next, we inferred the activity of AMPK by determining the phosphorylation levels of Thr172-AMPK. Notably, both oncogenes were capable of increasing phosphorylation of this residue (Fig. 1C). This kinase activation permitted us to conclude that the amount of ATP/AMP and NADP/ NADPH were modified in response to the 'oncogenic stress'.

The increase in LC3-II indicated differences in autophagy dynamics. Thus, we next analyzed how the RASv12transformed cells respond to a drug that induces mitochondrial stress, when compared with the parental cell lines. For this purpose, we used the MCF10A-GFP and MCF10A-RASv12 cells, as well as a breast tumor cell line (MCF7), transfected with both constructs (GFP and RASv12).

The cell lines containing GFP or RASv12, derived from either MCF10A or MCF7 cells, were treated with lis/lin/GO, that produced mitochondrial stress and consequently induced potent autophagy as previously described $(10,11)$. The lis/lin/GO treatment showed a marked growth inhibition in the MCF10A cells either untransformed (LV-GFP) (Fig. 2A) or transformed (LV-RASv12) (Fig. 2C) as determined by the MTT assay. Similar results were observed in the MCF7 and MCF7-RASv12 cells (Fig. 2B and D).

Notably, when we used a wide inhibitor of class III PI3K, 3-methyladenine (3MA), this effect was prevented or highly diminished only in the RAS-transformed cells in both cell types (Fig. 2C and D), whereas in the non-transformed cells the toxic effect generated by the $l i s /$ lin/GO system was not prevented (Fig. 2A and B).

Collectively, we conclude that the RAS-expressing cells were more sensitive to the inhibition of autophagy. To confirm this hypothesis, we determined whether the levels of p62, one of the main partners of LC3B $(17,18)$, is modified by the lis/ lin/GO treatment. Thus, we observed that either c-Myc or RAS transformation increased the amount of p62, and this protein level was reduced, probably by degradation, when autophagy was triggered by lis/lin/GO treatment in either the c-Myc- or Ras-expressing MCF10A cells (Fig. 3A).

There is a close relationship between metabolism and cellular damage generated by stress. In addition the mitochondrial toxic effect generated by the $l i s /$ lin/GO system permitted us to hypothesize the participation of AMPK in AMP/ATP deregulation. We showed that Myc or RAS transformation generated an increase in AMPK activity (Fig. 1C), and inhibition of AMPK by compound $\mathrm{C}(10 \mu \mathrm{M})$ partially recovered the cell survival reduced by lis/lin/GO treatment in the MCF10ARASv12 cells (Fig. 3B).

It has been previously reported that AMPK mediates FoxO3-positive regulation of energy balance and stress (9). This transcriptional factor controls the expression of an important set of genes related to autophagy, such as LC3B or BNIP3 among others, as reported in RAS-mediated senescence or in muscular atrophy $(4,19,20)$. Therefore, to validate the activation of AMPK due to these tumor transformations, we analyzed the expression of phosphorylated FoxO3 and the protein levels of LC3B and BNIP3. 

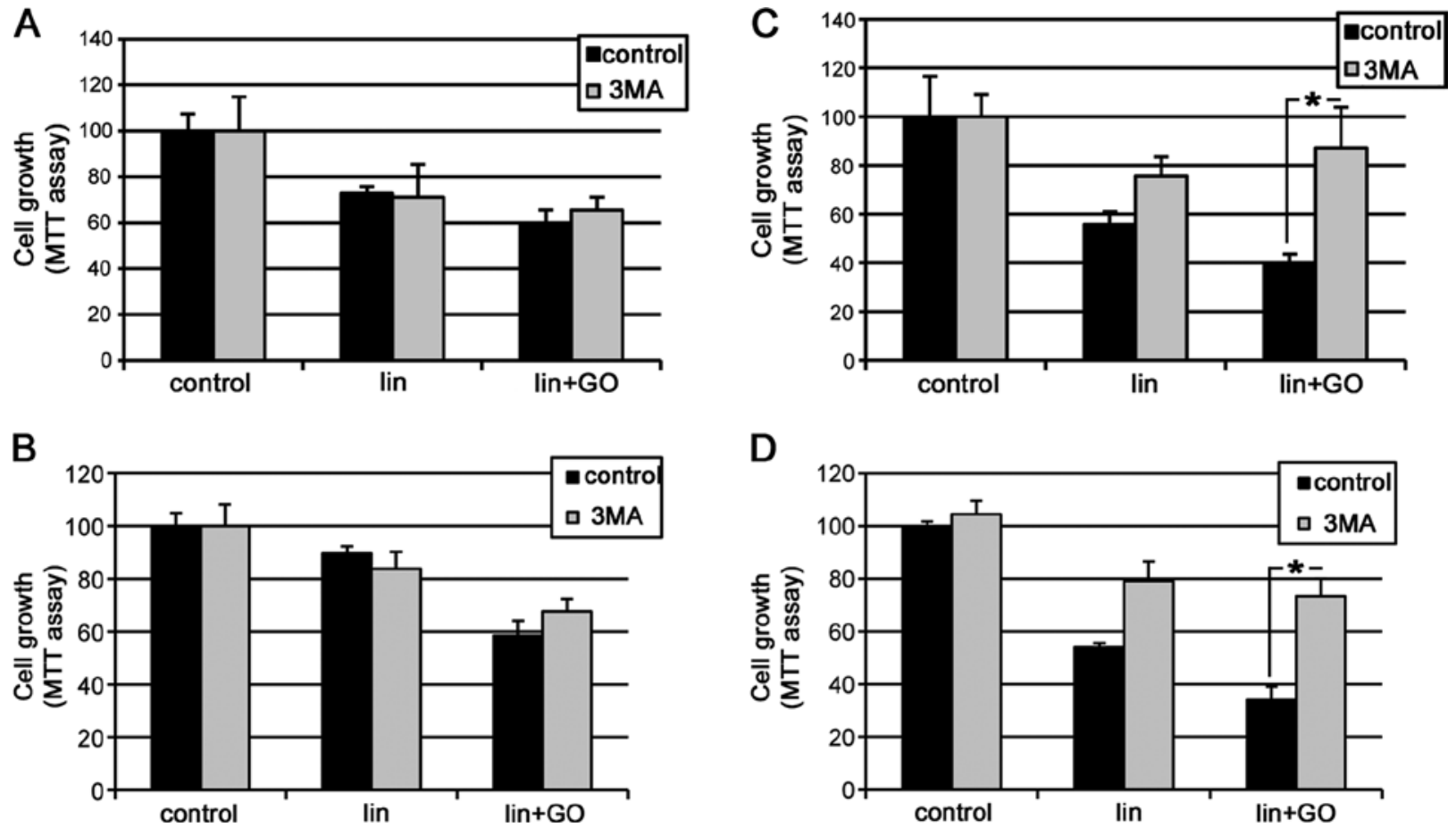

Figure 2. Cell transformation sensitizes cells to autophagy induction. (A-D) Cell growth/viability was determined by MTT assay in (A and C) MCF10A or (B and D) MCF7 cells, either in control conditions or when infected by Adeno-lis (MOI of 10) in the presence of lin $(0.5 \mathrm{mg} / \mathrm{ml}), \mathrm{GO}(5.5 \mathrm{mEU} / \mathrm{ml}) \mathrm{after} 48 \mathrm{~h}$, in the absence or presence of 3-methyladenine (3MA) (10 mM). Cell growth determination in MCF10A cells either when infected with (A) LV-GFP or with (B) LV-RASv12. Similar determination using MCF7 cells, after expression of (C) LV-control or (D) LV-RASv12, in the absence or presence of 3MA (10 mM). Graphs show the means \pm SD of 4 independent samples. * $<<0.05$. GO, glucose oxidase.

A

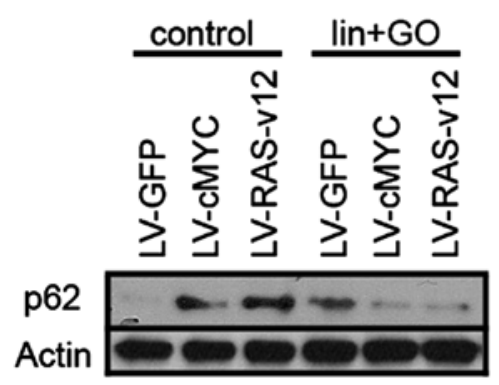

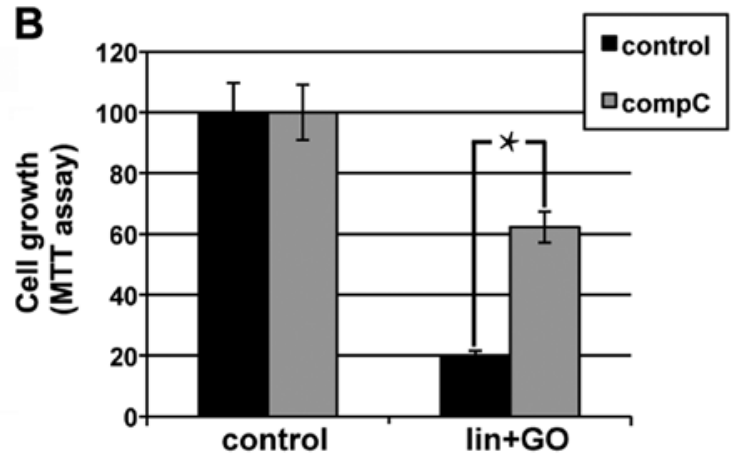

Figure 3. Regulation of p62 levels and involvement of AMPK in autophagy induced by the lis/lin/GO system. (A) Representative western blotting showing p62 levels in the GPF-, c-Myc- and RAS-v12-transduced MCF10A cells when infected with adenolis (MOI of 10) and treated with lin (0.5 mg/ml) and GO $(5.5 \mathrm{mEU} / \mathrm{ml})$ for $24 \mathrm{~h}$. (B) Cell growth measured by MTT assay of the MCF10A-RASv12 cells in the presence of compound C (compC; $10 \mu \mathrm{M})$ an inhibitor of AMPK, following infection with adenolis and treatment with lin and GO as in A for $36 \mathrm{~h}$. Graphs show the means \pm SD of 4 independent samples. ${ }^{*}<<.05$. GO, glucose oxidase.

Our data indicated that both c-Myc and RAS activated AMPK by increasing the level of phosphorylated AMPK $\alpha$ in Thr172 (pAMPK) (Fig. 4A); in parallel LC3-II was increased (Fig. 4A). This activation of AMPK was correlated with an increase in p-FoxO3-Ser413, coinciding with an increase in BNIP3 and LC3B (Fig. 4A). This AMPK kinase activation was confirmed since following incubation with the inhibitor compound $\mathrm{C}(10 \mu \mathrm{M})$ both FoxO3-Ser413 phosphorylation and BNIP3 and LC3B expression were severely reduced (Fig. 4A). The additional stress generated by the lis/lin/GO system increased p-AMPK-Thr172 as well as FoxO3-Ser413, which were correlated with an increase in BNIP3 expression and LC3-II (Fig. 4B) more markedly in the
c-Myc- and RAS-transformed cells. Similarly the addition of AMPK inhibitor, compound C, completely blocked FoxO3 phosphorylation, therefore preventing BNIP3 and LC3-II induction (Fig. 4A). Similarly to Compound C, the expression of dominant-negative version of AMPK (AMPK-DN) reduced the enhancement of LC3-II triggered by lin+GO (Fig. 4C).

To assess the involvement of $\mathrm{FoxO} 3$ in the regulation of $\mathrm{LC} 3$, we infected MCF10A-RASv12 cells with either a dominantnegative (dnFoxO3) or a constitutively active version of FoxO3 (caFoxO3), as well as an empty virus (pBabe) as a control. It was observed that $\mathrm{dnF} F \mathrm{OO} 3$ blocked the expression of $\mathrm{LC} 3$ as well as the amount of LC3-II (Fig. 5A); in contrast, the expression of caFoxO3 increased the level of LC3-II (Fig. 5A). 
A

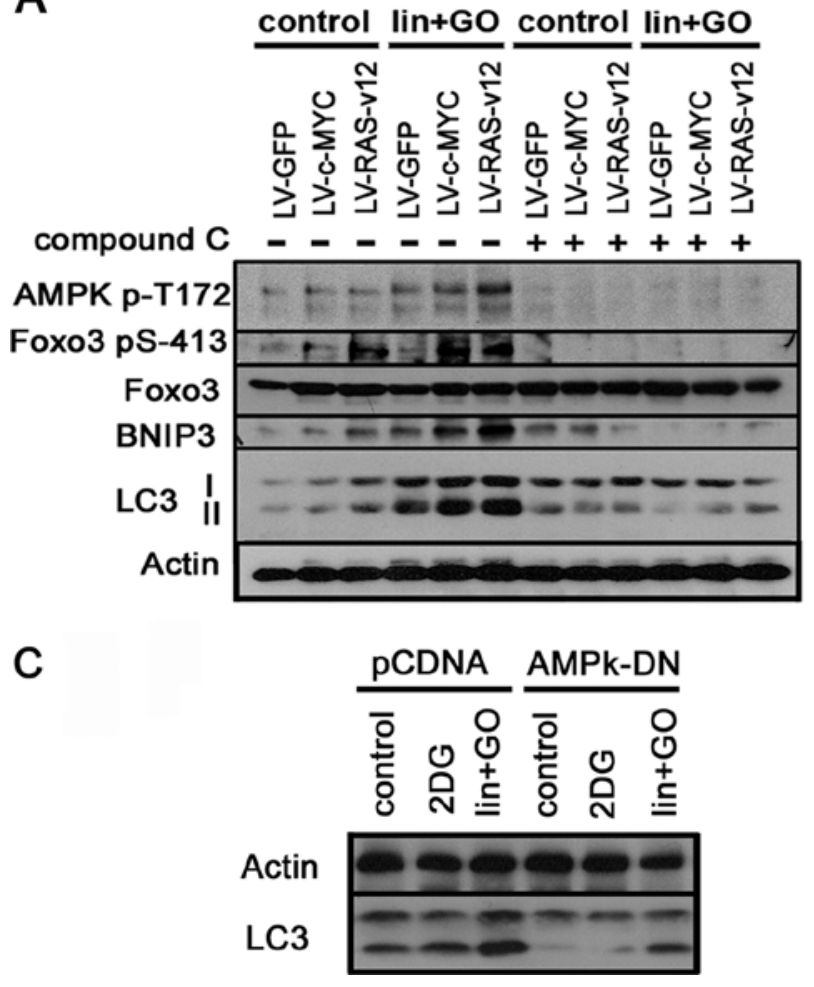

B
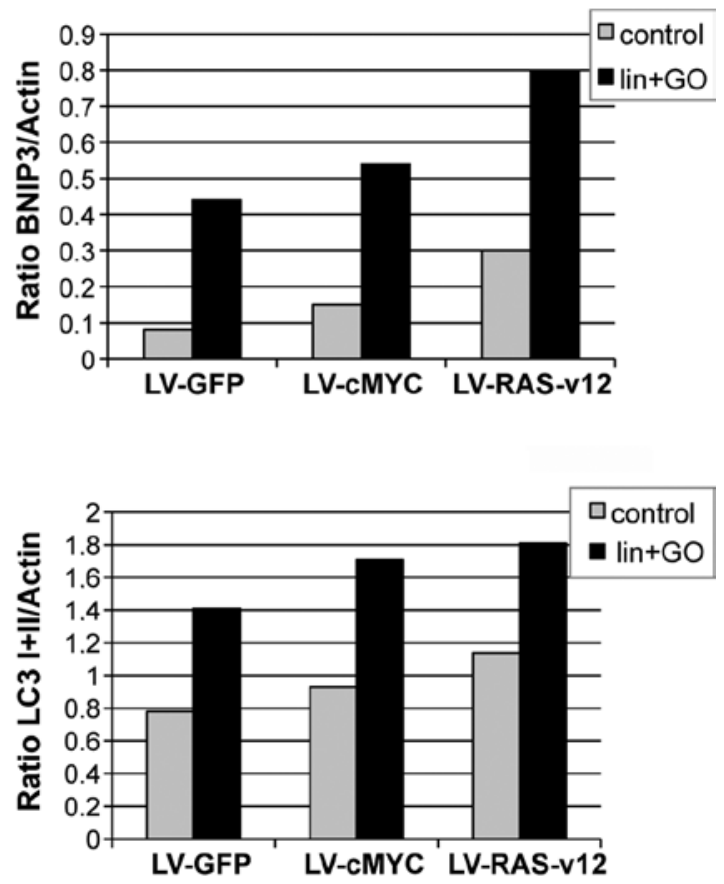

Figure 4. Role of the AMPK-FoxO3 axis in the sensitization to autophagy induced by RAS-v12. (A) Representative western blot analysis of phospho-AMPK and the subsequent phosphorylation of FoxO3 in ser413 as well as the upregulation of its targets BNIP3 and LC3B, in the MCF10A cells transduced with GPF, c-Myc, or RAS-v12. The transformed MCF10A and control cells (LV-GFP) were treated with or without $10 \mu \mathrm{M}$ of compound C; and additionally these cells were infected with adenolis and treated with lin and GO for $12 \mathrm{~h}(\mathrm{lin}+\mathrm{GO})$ or untreated (control). (B) Graphs represent quantification of the western blot analysis shown in A. GO, glucose oxidase. (C) The expression of dominant-negative version of AMPK (AMPK-DN) reduced the enhancement of LC3-II triggered by lin+GO.

A

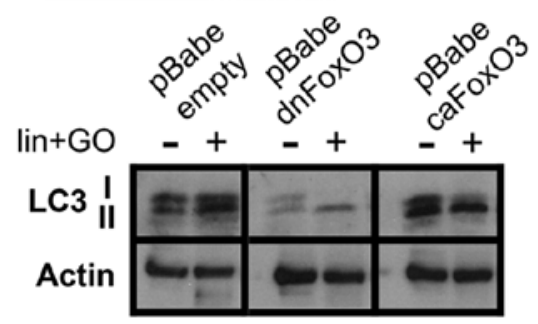

B

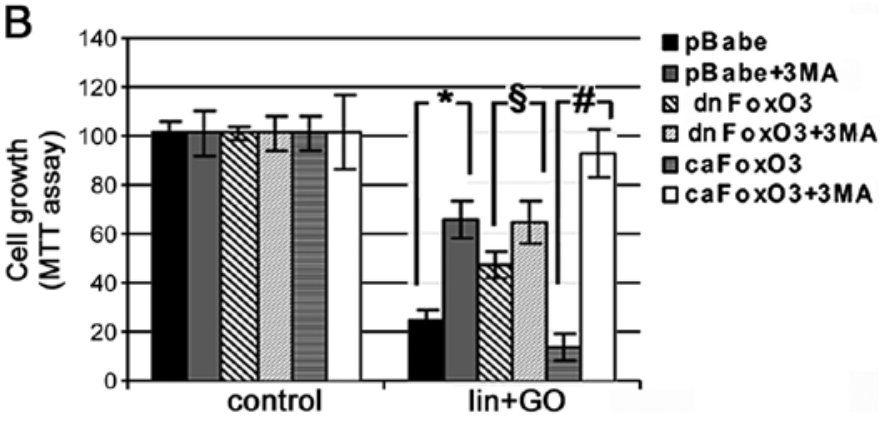

Figure 5. The role of FoxO3 in the sensitization to autophagy mediated by Ras. (A) Representative western blot analysis of LC3 levels from MCF10A-RASv12 cells infected with a control retrovector (pBabe-empty) or the same vector encoding a dominant-negative of FoxO3 (dnFoxO3), or a constitutively active form of FoxO3 (caFoxO3). The cells were then infected with adenolis (MOI of 10) and treated with lin (0.5 mg/ml) and GO (5.5 mEU/ml). (B) Cell growth was assessed by MTT assay of the cells described and treated as in A, in the absence or presence of 3-methyladenine (3MA) (10 mM). Graph shows the means \pm SD of 4 independent samples. ${ }^{*} \mathrm{p}<0.05 ;{ }^{\S} \mathrm{p}=0.2 ;{ }^{*} \mathrm{p}=0.0001$. GO, glucose oxidase.

Notably, the dnFoxO3 construct partially recovered the cell survival rate following lis/lin/GO treatment, while the caFoxO3 construct diminished the survival of the MCF10ARASv12 cells (Fig. 5B). The subsequent inhibition of class III PI3K by 3MA increased the survival of the control cells (infected by LV-GFP). Notably, the presence of 3MA increased the survival of the caFoxO3 cells even more than that in the control cells ( $p=0.045$ vs. $p=0.0001$ ) (Fig. $5 B$ ), whereas no additional increase was observed in the dnFoxO3 3MA-treated cells $(\mathrm{p}=0.2)$ (Fig. 5B). All these data suggest that autophagy induced by $l i s /$ lin/GO was mediated at least in part by FoxO3 activity.
Based on the fact that the $l i s /$ lin/GO treatment reduced the growth of MCF7-RASv12 cells, we aimed to ascertain whether this affects the growth capacity of the MCF7-RASv12 tumor cells in vivo. To address this issue, we inoculated MCF7RAS-lis cells into the flank of nude mice and carried out treatment with vehicle or $l i s /$ lin/GO compounds as described in Materials and methods. As shown in Fig. 6A, treatment with lis/lin/GO significantly reduced in vivo tumor growth from day 11 onwards. Additionally we analyzed the induction of autophagy (LC3-II levels) in the untreated and treated tumors. Western blot analyses of the samples $(n=4)$ showed that tumor extracts from the lis/lin/GO-treated tumors showed a substan- 
A

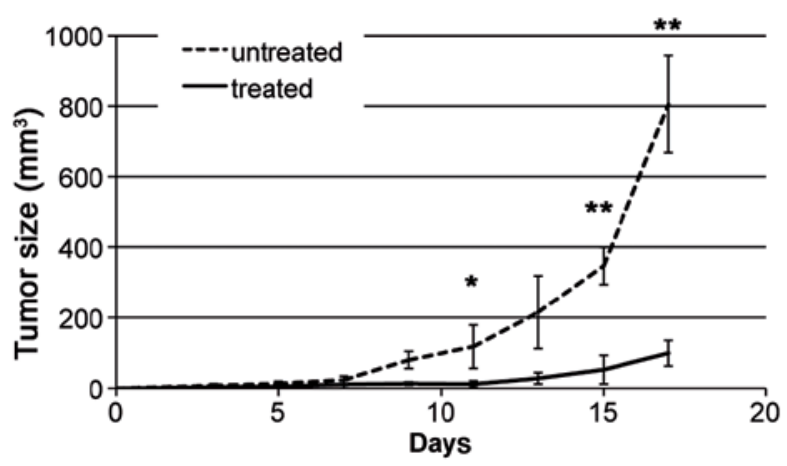

B

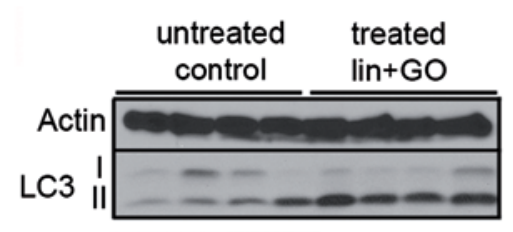

C

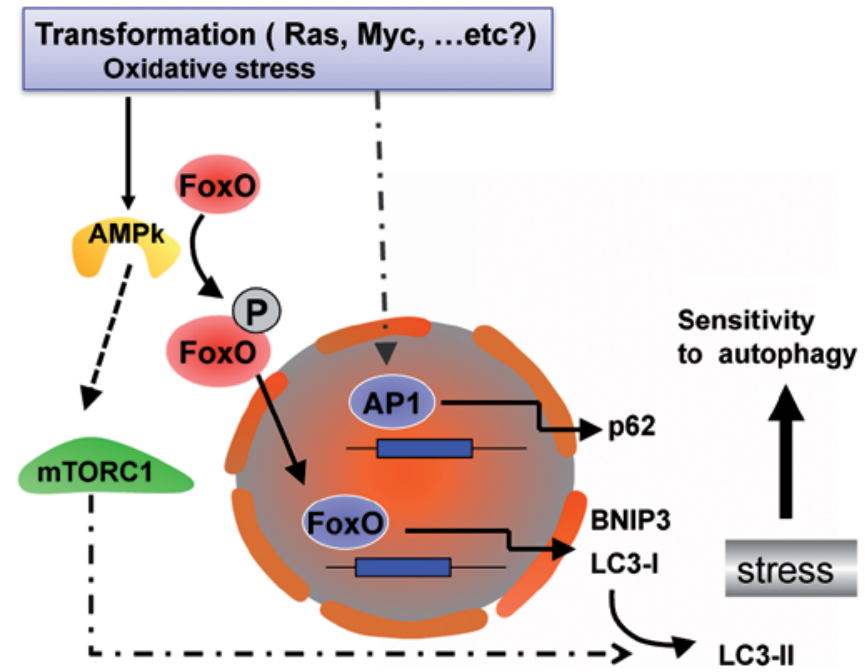

Figure 6. Tumor growth of the xenotransplanted MCF7-RASv12-lis cells in nude mice. (A) Animals were inoculated in their flanks with MCF7-RASv12-lis cells. Then the tumors in the right flank were injected daily with lin and GO, as described in Materials and methods, while the left ones were not treated. Graphs show the means and SE of the tumor volume in 6 mice/group. (B) Representative western blot analysis (from n=4 mice) of LC3 levels in the untreated and treated tumors. (C) Schematic illustration of the mechanism proposed to correlate oncogene transformation by c-Myc or RAS-v12; and the regulation and sensitivity to autophagy. The oncogenic transformation (RAS, c-Myc) via deregulation of AMPK and/or 'oncogenic stress' maintains a high level of basal autophagy, which becomes more sensitive to 'mitotoxic compounds'. (C) The expression of dominant-negative version of AMPK (AMPK-DN) reduced the enhancement of LC3-II triggered by lin+GO.

tial increase in levels of LC3-II when compared with these levels in the solvent-treated tumors (Fig. 6B).

In conclusion, transformation mediated by both oncogenes enhanced the sensitivity of the transformed cells to autophagy induction when compared with the untransformed cells. This may have therapeutic interest. This increase in sensitivity under a condition of potent mitochondrial stress is mediated, at least in part, by the AMPK-FoxO3 axis as summarized in the scheme in Fig. 6C.

\section{Discussion}

It is widely accepted that tumor cells modify autophagy generating a dependency on this process for maintaining survival and overcoming metabolic stress. Thus, tumor cells are sensitive to the blockage of autophagy $(14,21)$ as well as to its stimulation (22-24). It has been previously demonstrated that autophagy is necessary for activated Ras-mediated transformation (7). In the present study, we demonstrated that tumor processes driven by genes such as c-Myc or RAS (RAS-v12) $(14,16,25)$ sensitized cells to autophagy induction by the lis/lin/GO system. This system has been reported to generate mitochondrial stress-triggering autophagy $(10,11)$. Mitochondrial targeting is an attractive antitumor strategy due to the close relationship between oncogenic processes and the modification of the mitochondrial metabolic status $(26,27)$. Additionally, mitophagy is a mechanism capable of recycling damaged mitochondria, producing ATP (28) and reducing cellular stress. Therefore, a disturbance in this process can affect the proliferation and survival of tumor cells.

The present data confirmed that the $l i s /$ lin/GO system increased AMPK activity, as inferred from phosphorylation of FoxO3 and an increase in genes that are fundamental for autophagy induction such as BNIP3 and LC3B. These data confirm previous reports that indicate that AMPK favors the transcriptional activation of FoxO3 by the phosphorylation of its Ser413 or Ser588 residues (9), and this transcriptional factor controls the induction of BNIP3 and LC3B genes depending on cell type and context $(19,20)$.

In addition, our data demonstrated that the oncogenes, c-Myc and RAS, also modified AMPK activity by generating the upregulation of BNIP3 and LC3B. We demonstrated that the $l i s /$ lin/GO-induced autophagy activated this pathway, which was more evident in the RAS-transformed cells, and presented the highest levels of BNIP3 and LC3B in a clear correlation with the phosphorylation of FoxO3. Obviously, we cannot ignore the fact that other regulatory elements such as p38 may contribute to the final regulatory loop after transformation (29). In cases, transformed cells with or without $l i s /$ lin/GO treatment, the inhibitor of AMPK or the dominant-negative version of AMPK prevented the increase in LC3-II. Thus, these data allow the conclusion that autophagy is enhanced in c-Myc and RAS oncogenic models. A similar effect of enhanced autophagy was reported in cancer cells transformed by various tyrosine kinases such as EGFR or Abl (30-33). In these cases, tumor progression was associated with an enhancement of autophagy, dependent or independent, of tyrosine kinase activity. Thus, it can be proposed that oncogenic transformation which enhances autophagy is an important mechanism for tumor growth and progression.

Thus, we propose that a high percentage of tumors that bear oncogenes may have proliferative or metabolic stress which makes cells more sensitive to autophagy affecting tumor growth in vitro and in vivo. This susceptibility to the 
induction of autophagy is regulated, at least in certain cases, by the AMPK-FoxO pathway. Additionally, we hypothesize that autophagy overstimulation by mitochondrial stress induction can be considered as a therapeutic strategy against cancer.

\section{Acknowledgements}

We would like to thank Dr Clemens Schmitt, Dr Maria Soengas, Dr Patricia Boya and Dr Anne Brunet for providing the plasmids and antibodies. The laboratory of M.I. was supported by funds from the Fondo de Investigación Sanitaria, Ministerio de Sanidad y Consumo (PI06/0554), and from FINA Biotech. R.G. was supported by the Fundación Mario Losantos del Campo and Juan de la Cierva postdoctoral fellowship (Ministerio de Economía y Competitividad). The laboratory of F.W. was supported by grants from the Plan Nacional of the Dirección General de Ciencia y Tecnología (SAF2012-39148-C03-01), from the European Union (EU-FP7-2009-CT222887), and from CIBERNED (which is an initiative of ISCIII). The Centro de Biología Molecular S.O. is also the recipient of an institutional grant from the Ramón Areces Foundation.

\section{References}

1. Mathew R, Karp CM, Beaudoin B, Vuong N, Chen G, Chen HY, Bray K, Reddy A, Bhanot G, Gelinas C, et al: Autophagy suppresses tumorigenesis through elimination of p62. Cell 137: 1062-1075, 2009.

2. Tang YC, Williams BR, Siegel JJ and Amon A: Identification of aneuploidy-selective antiproliferation compounds. Cell 144: 499-512, 2011.

3. Courtois-Cox S, Genther Williams SM, Reczek EE, Johnson BW, McGillicuddy LT, Johannessen CM, Hollstein PE, MacCollin M and Cichowski K: A negative feedback signaling network underlies oncogene-induced senescence. Cancer Cell 10: 459-472, 2006.

4. Young AR, Narita M, Ferreira M, Kirschner K, Sadaie M, Darot JF, Tavaré S, Arakawa S, Shimizu S, Watt FM, et al: Autophagy mediates the mitotic senescence transition. Genes Dev 23: 798-803, 2009.

5. Rong Y, McPhee CK, Deng S, Huang L, Chen L, Liu M, Tracy K, Baehrecke EH, Yu L and Lenardo MJ: Spinster is required for autophagic lysosome reformation and mTOR reactivation following starvation. Proc Natl Acad Sci USA 108: 7826-7831, 2011

6. Narita M, Young AR, Arakawa S, Samarajiwa SA, Nakashima T, Yoshida S, Hong S, Berry LS, Reichelt S, Ferreira M, et al: Spatial coupling of mTOR and autophagy augments secretory phenotypes. Science 332: 966-970, 2011.

7. Guo JY, Chen HY, Mathew R, Fan J, Strohecker AM, Karsli-Uzunbas G, Kamphorst JJ, Chen G, Lemons JM, Karantza V, et al: Activated Ras requires autophagy to maintain oxidative metabolism and tumorigenesis. Genes Dev 25: 460-470, 2011.

8. Flier JS, Mueckler MM, Usher P and Lodish HF: Elevated levels of glucose transport and transporter messenger RNA are induced by ras or src oncogenes. Science 235: 1492-1495, 1987.

9. Greer EL, Oskoui PR, Banko MR, Maniar JM, Gygi MP, Gygi SP and Brunet A: The energy sensor AMP-activated protein kinase directly regulates the mammalian FOXO3 transcription factor. J Biol Chem 282: 30107-30119, 2007.

10. García-Escudero V and Gargini R: Autophagy induction as an efficient strategy to eradicate tumors. Autophagy 4: 923-925, 2008.

11. Gargini R, García-Escudero V and Izquierdo M: Therapy mediated by mitophagy abrogates tumor progression. Autophagy 7: 466-476, 2011

12. Nikiforov MA, Riblett M, Tang WH, Gratchouck V, Zhuang D, Fernandez Y, Verhaegen M, Varambally S, Chinnaiyan AM, Jakubowiak AJ, et al: Tumor cell-selective regulation of NOXA by c-MYC in response to proteasome inhibition. Proc Natl Acad Sci USA 104: 19488-19493, 2007.

13. Beauséjour CM, Krtolica A, Galimi F, Narita M, Lowe SW, Yaswen P and Campisi J: Reversal of human cellular senescence: Roles of the p53 and p16 pathways. EMBO J 22: 4212-4222, 2003.
14. Amaravadi RK, Yu D, Lum JJ, Bui T, Christophorou MA, Evan GI, Thomas-Tikhonenko A and Thompson CB: Autophagy inhibition enhances therapy-induced apoptosis in a Myc-induced model of lymphoma. J Clin Invest 117: 326-336, 2007.

15. Thibodeaux CA, Liu X, Disbrow GL, Zhang Y, Rone JD, Haddad BR and Schlegel R: Immortalization and transformation of human mammary epithelial cells by a tumor-derived Myc mutant. Breast Cancer Res Treat 116: 281-294, 2009.

16. Datta S, Hoenerhoff MJ, Bommi P, Sainger R, Guo WJ, Dimri M, Band H, Band V, Green JE and Dimri GP: Bmi-1 cooperates with $\mathrm{H}$-Ras to transform human mammary epithelial cells via dysregulation of multiple growth-regulatory pathways. Cancer Res 67: 10286-10295, 2007.

17. Pankiv S, Clausen TH, Lamark T, Brech A, Bruun JA, Outzen H, Øvervatn A, Bjørkøy G and Johansen T: p62/SQSTM1 binds directly to Atg8/LC3 to facilitate degradation of ubiquitinated protein aggregates by autophagy. J Biol Chem 282: 24131-24145, 2007.

18. Duran A, Linares JF, Galvez AS, Wikenheiser K, Flores JM, Diaz-Meco MT and Moscat J: The signaling adaptor p62 is an important NF-kappaB mediator in tumorigenesis. Cancer Cell 13: 343-354, 2008.

19. Mammucari C, Milan G, Romanello V, Masiero E, Rudolf R, Del Piccolo P, Burden SJ, Di Lisi R, Sandri C, Zhao J, et al: FoxO3 controls autophagy in skeletal muscle in vivo. Cell Metab 6: 458-471, 2007.

20. Zhao J, Brault JJ, Schild A, Cao P, Sandri M, Schiaffino S, Lecker SH and Goldberg AL: FoxO3 coordinately activates protein degradation by the autophagic/lysosomal and proteasomal pathways in atrophying muscle cells. Cell Metab 6: 472-483, 2007.

21. Maclean KH, Dorsey FC, Cleveland JL and Kastan MB: Targeting lysosomal degradation induces p53-dependent cell death and prevents cancer in mouse models of lymphomagenesis. J Clin Invest 118: 79-88, 2008.

22. Kanzawa T, Zhang L, Xiao L, Germano IM, Kondo Y and Kondo S: Arsenic trioxide induces autophagic cell death in malignant glioma cells by upregulation of mitochondrial cell death protein BNIP3. Oncogene 24: 980-991, 2005.

23. Chang CP, Yang MC, Liu HS, Lin YS and Lei HY: Concanavalin A induces autophagy in hepatoma cells and has a therapeutic effect in a murine in situ hepatoma model. Hepatology 45: 286-296, 2007.

24. García-Escudero V, Gargini R and Izquierdo M: Glioma regression in vitro and in vivo by a suicide combined treatment. Mol Cancer Res 6: 407-417, 2008.

25. Kim H, Farris J, Christman SA, Kong BW, Foster LK, O'Grady SM and Foster DN: Events in the immortalizing process of primary human mammary epithelial cells by the catalytic subunit of human telomerase. Biochem J 365: 765-772, 2002

26. Levine AJ and Puzio-Kuter AM: The control of the metabolic switch in cancers by oncogenes and tumor suppressor genes. Science 330: 1340-1344, 2010.

27. Jeon SM, Chandel NS and Hay N: AMPK regulates NADPH homeostasis to promote tumour cell survival during energy stress. Nature 485: 661-665, 2012.

28. Dang CV: Links between metabolism and cancer. Genes Dev 26 : 877-890, 2012

29. Lin A, Yao J, Zhuang L, Wang D, Han J, Lam EW and Gan B: The FoxO-BNIP3 axis exerts a unique regulation of mTORC1 and cell survival under energy stress. Oncogene 33: 3183-3194, 2014.

30. Yogalingam G and Pendergast AM: Abl kinases regulate autophagy by promoting the trafficking and function of lysosomal components. J Biol Chem 283: 35941-35953, 2008.

31. Turcotte S, Chan DA, Sutphin PD, Hay MP, Denny WA and Giaccia AJ: A molecule targeting VHL-deficient renal cell carcinoma that induces autophagy. Cancer Cell 14: 90-102, 2008.

32. Weihua Z, Tsan R, Huang WC, Wu Q, Chiu CH, Fidler IJ and Hung MC: Survival of cancer cells is maintained by EGFR independent of its kinase activity. Cancer Cell 13: 385-393, 2008.

33. Lu Z, Luo RZ, Lu Y, Zhang X, Yu Q, Khare S, Kondo S, Kondo Y, Yu Y, Mills GB, et al: The tumor suppressor gene ARHI regulates autophagy and tumor dormancy in human ovarian cancer cells. J Clin Invest 118: 3917-3929, 2008. 